\title{
A new memetic algorithm for solving split delivery vehicle routing problem
}

\author{
Ali Bozorgi-Amiri ${ }^{\mathrm{a}^{*}}$, Vahid Mahmoodian ${ }^{\mathrm{b}}$, Elham Fahimnia ${ }^{\mathrm{c}}$ and Hamid Saffari ${ }^{\mathrm{b}}$
}

${ }^{a}$ School of Industrial Engineering, College of Engineering, University of Tehran, Tehran, Iran ${ }^{b}$ Department of Industrial Engineering, Iran University of Science and Technology, Tehran, Iran ${ }^{c}$ Department of Electrical Engineering, Amirkabir University, Tehran, Iran

\section{H R O N I C L E}

\begin{tabular}{l}
\hline Article history: \\
Received March 25, 2015 \\
Received in revised format \\
August 62015 \\
Accepted August 212015 \\
Available online \\
August 292015 \\
\hline Keywords: \\
SDVRP \\
Split delivery vehicle routing \\
problem \\
Memetic \\
\hline
\end{tabular}

\begin{abstract}
A B S T R A C T
Split delivery vehicle routing problem is one of the traditional types of routing problems in which the demand of different points can be divided among vehicles and the objective is to minimize the path length, which vehicles travel. In this paper, fuel cost of vehicles which is assumed to be dependent on their traveled path and load is considered as the objective functions. Namely, the cost of the consumed fuel is proportionate to the unit of load carried per unit of distance. In order to solve the proposed model a new memetic algorithm is developed which has two rows. The performance of the proposed algorithm for 21 standard problems is compared with the optimum solutions obtained from mathematical programming standard solver and the solutions of the same algorithm with single row solution representation. The results express the efficiency of developed algorithm.
\end{abstract}

\section{Introduction}

Split delivery vehicle routing problem is one of the traditional types of routing problems in which the demand of different points can be divided among vehicles and the objective is to minimize the path length, which vehicles travel (Archetti et al., 2006; Anh, 2014). By splitting demand among different vehicles, the cost of transportation could be reduced significantly. The idea of split delivery was first introduced by Dror and Trudeau $(1989,1990)$ and despite simplicity there appears to be many applications for this problem (Chen et al., 2007). There are also several solution strategies for solving this problem. Jin et al. (2008), for instance, presented a column generation approach for the Split delivery vehicle routing problem (SDVRP). Stålhane et al. (2012) offered a branch-price-and-cut method for a ship routing and scheduling problem with split loads. Belenguer et al. (2000) presented a lower bound for the split delivery vehicle routing problem and Moreno et al. (2010) improved the lower bounds for the split delivery vehicle routing problem. Archetti et al. (2006) presented a tabu search algorithm for the split delivery vehicle routing problem. Archetti and Speranza (2012) considered vehicle routing problems with split deliveries. Archetti et al. (2014) presented two exact branch-andcut algorithms for the SDVRP based on two relaxed formulations that provide lower bounds to the optimum. Boudia et al. (2007) offered an effective memetic algorithm with population management for * Corresponding author. Tel: +989126055245

E-mail address: alibozorgi@ut.ac.ir (A. Bozorgi-Amiri) 
the split delivery vehicle routing problem. Mota et al. (2007) proposed a metaheuristic for the vehicle routing problem with split demand, which was for the special case of the Vehicle Routing Problem in which the demands of the clients was split, i.e., any client can be serviced by more than one vehicle. Archetti et al. (2008) developed an optimization-based heuristic for the SDVRP and Aleman (2010) introduced an adaptive memory algorithm for this problem. Aleman and Hill (2010) offered a tabu search with vocabulary building method for the vehicle routing problem with split demands. Derigs et al. (2010) and Wen et al. (2015) presented a local search-based metaheuristics for the SDVRP. Wilck IV et al. (2012) offered a genetic algorithm for the split delivery vehicle routing problem while Khmelev and Kochetov (2015) built a hybrid VND method for this problem. Zhang et al. (2012) studied SDVRP for emergency logistics and proposed an evolutionary heuristic approach to solve such problem. Wang et al. (2013) performed a survey on vehicle routing problem by considering simultaneous deliveries and pickups with split loads and time windows. Wang et al. (2014) considered SDVRP for another application and using two-stage heuristic methods, they investigated vehicle routing problem with split deliveries and pickups. Yuyan et al. (2013) considered different perspective of SDVRP problem. Chen et al. (2014) performed a comprehensive survey on model and algorithm for an unpaired pickup and delivery vehicle routing problem with split loads. Zeng et al. (2014) presented an ant colony algorithm with memory grouping list for multi-depot vehicle routing problem. Zhang et al. (2015) presented an efficient forest-based tabu search algorithm for the SDVRP problem.

\section{The proposed study}

Split delivery vehicle routing problem is one of the traditional types of routing problems in which the demand of different points can be divided among vehicles and the objective is to minimize the path length, which vehicles travel. In this paper, fuel cost of vehicles which is assumed to be dependent on their traveled path and load is considered as the objective functions. Namely, the cost of the consumed fuel is proportionate to the unit of load carried per unit of distance. In order to solve the proposed model a new memetic algorithm is developed which has two rows to guarantee the optimality of solutions. Split delivery vehicle routing problem (SDVRP) can be defined as a graph of $\mathrm{G}=(\mathrm{V}, \mathrm{E})$ where $V=$ $\{0,1, \ldots, n\}$ represents the nodes and $E$ represents the arcs. Node 0 represents the origin and the other nodes represent demands. $D_{i j}$ denotes the length of $\operatorname{arc}(i, j) \in E, C_{1}$ and $C_{2}$ represent cost of travelling empty and full load vehicles, respectively. Therefore, a half load vehicles cost a linear combination of $C_{1}$ and $C_{2}$. Moreover, $\mathrm{d}_{\mathrm{i}}$ is the distance for demand $i \in V-\{0\}$, and there are $K$ vehicles with a capacity of $Q$.

Indices

$i, j \quad$ demands and depot point

$v \quad$ vehicles with $v \in\{1,2, \ldots, K\}$

$D_{i j} \quad$ distance between two point $(i, j) \in E$

$d_{i} \quad$ demand for node $i$

\section{Variables}

$x_{i j}^{v} \quad 1$ if vehicle $v$ moves from node $i$ to node $j$ and 0 , otherwise,

$y_{i v} \quad$ the amount of demand $j$, which is covered by vehicle $v$,

$z_{i j}^{v} \quad$ the amount of load $v$ for path $i-j$

The mathematical model can be represented as follows, 
$\min \quad C_{1} \sum_{v=1}^{K} \sum_{i, j \in V} D_{i j} x_{i j}^{v}+\frac{C_{2}-C_{1}}{Q} \sum_{v=1}^{K} \sum_{i, j \in V} D_{i j} z_{i j}^{v}$

subject to

$\sum_{i \in V} \sum_{v=1}^{K} x_{i j}^{v} \geq 1$,

$j \in V$

$\sum_{i \in V} x_{i j}^{v}-\sum_{i \in V} x_{j i}^{v}=0$

$j \in V ; v=1,2, \ldots, K$

$\sum_{i \in S} \sum_{j \in S} x_{i j}^{v} \leq|S|-1$

$v=1,2, \ldots, K$;

$S \subseteq V-\{0\}$

$y_{i v} \leq d_{i} \sum_{j \in V} x_{i j}^{v}$

$i \in V-\{0\}$;

$v=1,2, \ldots, K$

$\sum_{v=1}^{K} y_{i v}=d_{i}$

$i \in V-\{0\}$

$z_{i j}^{v} \leq Q x_{i j}^{v}$

$i \in V, j \in V-\{0\}$;

$v=1,2, \ldots, K$

$\sum_{j \in V} z_{j i}^{v}-\sum_{j \in V} z_{i j}^{v}=y_{i v}$

$i \in V-\{0\}$;

$v=1,2, \ldots, K$

$x_{i j}^{v} \in\{0,1\}$,

$i, j \in V ; v=1,2, \ldots, K$

$$
y_{i v} \geq 0, z_{i j}^{v} \geq 0
$$

$i, j \in V ; v=1,2, \ldots, K$

The objective function of this model minimizes total cost of transportation. Eq. (2) guarantees that each node is visited at least once. Eq. (3) is associated with the flow between two nodes. Eq. (4) prevents the possibility of having circle among nodes. Eq. (5) is to ensure that each node is visited by a vehicle when there is a service delivered. Eq. (6) and Eq. (7) represent demand and supply constraints, respectively. Eq. (8) is associated with the amount of demand delivered by each vehicle. Finally, other variables determine the type of variables.

The proposed study of this paper uses memetic algorithm (Golberg, 1989; Back et al., 1997; Merz \& Freisleben, 2001) for determine the near optimal solution for the proposed study of this paper. Fig. 1 demonstrates the Chromosome used for this study.

\begin{tabular}{c|c|c|c|c|c|}
1 & 2 & 3 & $\ldots$ & $n-1$ & $n$ \\
\hline 0.45827 & 0.79874 & 0.87947 & $\ldots$ & 0.48258 & 0.63179 \\
\hline 0.28666 & 0.74006 & 0.38395 & $\ldots$ & 0.19846 & 0.82163 \\
\hline
\end{tabular}

Fig. 1. The structure of the chromosome

According to Fig. 1, there are two columns where the first column demonstrates the priority of assigning demands for different routes and the second row shows the priority of meeting various demand points. The proposed study uses random numbers, which eliminates the possibility of having infeasible solutions. The local search for the proposed study of this paper is as follows, 


\section{Algorithm 1}

1. Setup parameters,

2. Generate the initial population randomly,

3. Choose a member of population with the best fitness,

4. Until termination criteria is reached do the following,

4.1 Generate parents

4.2 Generate children using mutation operation on parents,

4.3 Choose $10 \%$ of the population and do a local search,

4.4 Evaluate the population,

4.5 Choose the members with the best fitness,

4.6 Choose the next generation.

\section{The results}

In this section, we present the implementation of the proposed method of this paper using some benchmark problems introduced by Chen et al. (2007). The method has been coded in MATLAB software. The results are shown when we use one column or two columns method to represent the initial solution. In addition, the mathematical model has been coded in GAMS software package and it was run for small scale problems and the solutions were compared with near-optimal solutions obtained using memetic method. Table 1 shows the results of our implementation.

\section{Table 1}

The summary of the implementation of memetic method versus GAMS method

\begin{tabular}{|c|c|c|c|c|c|c|c|c|c|}
\hline \multirow{2}{*}{ Problem } & \multicolumn{3}{|c|}{ One column } & \multicolumn{3}{|c|}{ Two column } & \multicolumn{2}{|c|}{ Optimal solution } & \multirow{2}{*}{$\begin{array}{c}\text { Difference } \\
\text { (\%) }\end{array}$} \\
\hline & Mean & Best & Time & Mean & Best & Time & Obj- func. & Time Cpu & \\
\hline 1 & 261.6 & 261.6 & 0.0571 & 261.6 & 261.6 & 0.129 & 261.6 & 0.34 & 0 \\
\hline 2 & 353.4 & 353.4 & 0.0393 & 347.8 & 347.8 & 1.4731 & 347.8 & 0.59 & 0 \\
\hline 3 & 278.2 & 278.2 & 0.375 & 278.2 & 278.2 & 1.7874 & 278.2 & 0.56 & 0 \\
\hline 4 & 333.4 & 333.4 & 0.6375 & 328.6 & 328.6 & 2.0253 & 328.6 & 0.75 & 0 \\
\hline 5 & 407.5 & 407.5 & 0.4449 & 410.5 & 403.9 & 4.256 & 403.9 & 0.94 & 0 \\
\hline 6 & 328.5 & 327.6 & 0.8593 & 319.2 & 319.2 & 7.3423 & 319.2 & 0.89 & 0 \\
\hline 7 & 372.6 & 372.6 & 0.7904 & 380.2 & 372.6 & 1.9636 & 372.6 & 0.94 & 0 \\
\hline 8 & 514.7 & 510.6 & 0.7682 & 512.4 & 500.4 & 5.4767 & 500.4 & 1.66 & 0 \\
\hline 9 & 417.4 & 389.35 & 0.7688 & 386.3 & 384.71 & 5.6282 & 384.71 & 3.12 & 0 \\
\hline 10 & 438.13 & 437.8 & 1.0168 & 435.93 & 426.2 & 3.3591 & 426.2 & 3.54 & 0 \\
\hline 11 & 391.95 & 381.85 & 1.8452 & 390.35 & 381.85 & 3.7663 & 381.85 & 1.43 & 0 \\
\hline 12 & 482.17 & 475.1 & 2.8398 & 475.63 & 455.9 & 4.151 & 455.9 & 6.26 & 0 \\
\hline 13 & 459.03 & 450.3 & 2.8022 & 457.3 & 451.7 & 6.5858 & 435.1 & 9.38 & 3.82 \\
\hline 14 & 515.47 & 499.2 & 2.7051 & 502.27 & 497.2 & 7.6222 & 497.2 & 25.62 & 0 \\
\hline 15 & 430.03 & 421.7 & 2.408 & 423.77 & 408.5 & 8.0459 & 408.5 & 40.67 & 0 \\
\hline 16 & 489.2 & 484.7 & 3.0733 & 484.6 & 472.1 & 8.0318 & 459.65 & 184.92 & 2.71 \\
\hline 17 & 502.52 & 498.83 & 2.3698 & 497.22 & 495.05 & 7.0091 & 471.53 & 233.78 & 4.99 \\
\hline 18 & 563.4 & 543.1 & 3.229 & 2136.33 & 516.9 & 6.5164 & 513.9 & 348.76 & 0.58 \\
\hline 19 & 604.26 & 593.31 & 4.0662 & 593.92 & 584.21 & 8.0763 & 581.79 & 1738.04 & 0.42 \\
\hline 20 & 695.69 & 662.2 & 5.1335 & 682.61 & 656.14 & 13.9904 & 630.2 & 2514.91 & 4.12 \\
\hline 21 & 831.82 & 793.79 & 5.1035 & 817.9 & 776.77 & 14.552 & $738.67^{*}$ & 7200 & 5.16 \\
\hline Mean & 460.52 & 451.24 & 1.9682 & 529.65 & 443.86 & 5.7994 & 422.94 & 586.53 & 1.05 \\
\hline
\end{tabular}

Note that the best solution obtained in 7200 seconds could be non-optimal. In addition, Fig. 1 shows the results of our proposed method for the best and average solutions.

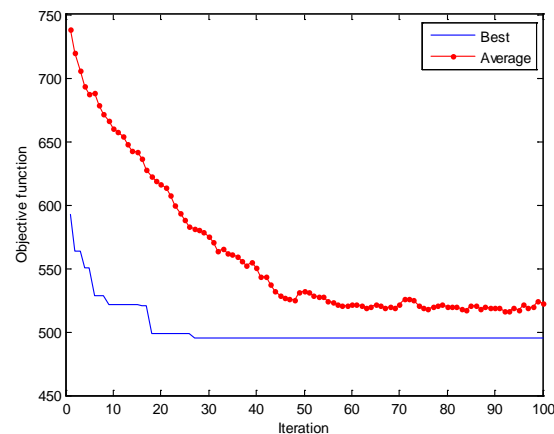

Fig. 1. The best versus average solution 


\section{Conclusion}

In this paper, we have presented a memetic problem for SDVRP problem where the fuel cost of vehicles which were assumed to be dependent on their traveled path and load was considered as the objective functions. Namely, the cost of the consumed fuel is proportionate to the unit of load carried per unit of distance. In order to solve the proposed model a new memetic algorithm has been developed which contained two rows to guarantee the optimality of solutions. The performance of the proposed algorithm for 21 standard problems is compared with the optimum solutions obtained from mathematical programming standard solver and the solutions of the same algorithm with single row solution representation. The results have stated the efficiency of developed algorithm.

\section{Acknowledgement}

The authors would like to thank the anonymous referees for constructive comments on earlier version of this paper.

\section{References}

Aleman, R. E., Zhang, X., \& Hill, R. R. (2010). An adaptive memory algorithm for the split delivery vehicle routing problem. Journal of Heuristics, 16(3), 441-473.

Aleman, R. E., \& Hill, R. R. (2010). A tabu search with vocabulary building approach for the vehicle routing problem with split demands. International Journal of Metaheuristics, 1(1), 55-80.

Anh, M. T. Q. (2014). Tabu search heuristic for vehicle routing problem with split delivery (Doctoral dissertation, International University HCMC, Vietnam).

Archetti, C., Savelsbergh, M. W., \& Speranza, M. G. (2006). Worst-case analysis for split delivery vehicle routing problems. Transportation Science,40(2), 226-234.

Archetti, C., Speranza, M. G., \& Hertz, A. (2006). A tabu search algorithm for the split delivery vehicle routing problem. Transportation Science, 40(1), 64-73.

Archetti, C., Speranza, M. G., \& Savelsbergh, M. W. (2008). An optimization-based heuristic for the split delivery vehicle routing problem. Transportation Science, 42(1), 22-31.

Archetti, C., \& Speranza, M. G. (2012). Vehicle routing problems with split deliveries. International transactions in operational research, 19(1-2), 3-22.

Archetti, C., Bianchessi, N., \& Speranza, M. G. (2014). Branch-and-cut algorithms for the split delivery vehicle routing problem. European Journal of Operational Research, 238(3), 685-698.

Back, T., Fogel, D. B., \& Michalewicz, Z. (1997). Handbook of evolutionary computation. IOP Publishing Ltd..

Belenguer, J. M., Martinez, M. C., \& Mota, E. (2000). A lower bound for the split delivery vehicle routing problem. Operations Research, 48(5), 801-810.

Boudia, M., Prins, C., \& Reghioui, M. (2007). An effective memetic algorithm with population management for the split delivery vehicle routing problem. InHybrid Metaheuristics (pp. 16-30). Springer Berlin Heidelberg.

Chen, S., Golden, B., \& Wasil, E. (2007). The split delivery vehicle routing problem: Applications, algorithms, test problems, and computational results.Networks, 49(4), 318-329.

Chen, Q., Li, K., \& Liu, Z. (2014). Model and algorithm for an unpaired pickup and delivery vehicle routing problem with split loads. Transportation Research Part E: Logistics and Transportation Review, 69, 218-235.

Derigs, U., Li, B., \& Vogel, U. (2010). Local search-based metaheuristics for the split delivery vehicle routing problem. Journal of the Operational Research Society, 61(9), 1356-1364.

Dror, M., \& Trudeau, P. (1989). Savings by split delivery routing. Transportation Science, 40, 141-145.

Dror, M., \& Trudeau, P. (1990). Split delivery routing. Naval Research Logistics (NRL), 37(3), 383402. 
Golberg, D. E. (1989). Genetic algorithms in search, optimization, and machine learning. Addion wesley, 1989.

Jin, M., Liu, K., \& Eksioglu, B. (2008). A column generation approach for the split delivery vehicle routing problem. Operations Research Letters, 36(2), 265-270.

Khmelev, A., \& Kochetov, Y. (2015). A hybrid VND method for the split delivery vehicle routing problem. Electronic Notes in Discrete Mathematics, 47, 5-12.

Merz, P., \& Freisleben, B. (2001). Memetic algorithms for the traveling salesman problem. Complex Systems, 13(4), 297-346.

Moreno, L., De AragãO, M. P., \& Uchoa, E. (2010). Improved lower bounds for the split delivery vehicle routing problem. Operations Research Letters, 38(4), 302-306.

Mota, E., Campos, V., \& Corberán, Á. (2007). A new metaheuristic for the vehicle routing problem with split demands. In Evolutionary Computation in Combinatorial Optimization (pp. 121-129). Springer Berlin Heidelberg.

Stålhane, M., Andersson, H., Christiansen, M., Cordeau, J. F., \& Desaulniers, G. (2012). A branchprice-and-cut method for a ship routing and scheduling problem with split loads. Computers \& Operations Research, 39(12), 3361-3375.

Wang, Y., Ma, X., Lao, Y., Wang, Y., \& Mao, H. (2013). Vehicle routing problem: simultaneous deliveries and pickups with split loads and time windows. Transportation Research Record: Journal of the Transportation Research Board, (2378), 120-128.

Wang, Y., Ma, X. L., Lao, Y. T., Yu, H. Y., \& Liu, Y. (2014). A two-stage heuristic method for vehicle routing problem with split deliveries and pickups.Journal of Zhejiang University SCIENCE C, 15(3), 200-210.

Wen, Z. Z., Dong, X. Y., \& Han, S. (2015, June). An iterated local search for the Split Delivery Vehicle Routing Problem. In International Conference on Computer Information Systems and Industrial Applications. Atlantis Press.

Wilck IV, J. H., \& Cavalier, T. M. (2012). A genetic algorithm for the split delivery vehicle routing problem. American Journal of Operations Research, 2(2), 10.

Yuyan, M., Jiafu, T., \& Jing, G. (2013, July). Split delivery weighted vehicle routing problem: comparison perspective. In Control Conference (CCC), 2013 32nd Chinese (pp. 8436-8440). IEEE.

Zeng, W., He, Y. L., \& Zheng, X. J. (2014, July). An ant colony algorithm with memory grouping list for multi-depot vehicle routing problem. In Advanced Materials Research (Vol. 926, pp. 33543358).

Zhang, J., Peng, J., Xu, Z., \& Xu, W. (2012, March). SDVRP model for emergency logistics and evolutionary heuristic approach. In Automatic Control and Artificial Intelligence (ACAI 2012), International Conference on (pp. 1809-1812). IET.

Zhang, Z., He, H., Luo, Z., Qin, H., \& Guo, S. (2015, April). An efficient forest-based tabu search algorithm for the Split-delivery Vehicle Routing Problem. InTwenty-Ninth AAAI Conference on Artificial Intelligence. 